\title{
The 'magic' of magic camp from the perspective of children with hemiparesis
}

\author{
Kevin Spencer', Hon K. Yuen ${ }^{2 *}$, Gavin R. Jenkins ${ }^{2}$, Kimberly Kirklin ${ }^{3}$, Laura K. Vogtle ${ }^{2}$, Drew Davis ${ }^{4}$ \\ 'Department of Education, Carlow University, Pittsburgh, PA, USA \\ ${ }^{2}$ Department of Occupational Therapy, University of Alabama at Birmingham, Birmingham, AL, USA \\ ${ }^{3} U A B$ Institute for Arts in Medicine, University of Alabama at Birmingham, Birmingham, AL, USA \\ ${ }^{4}$ Division of Pediatric Rehabilitation Medicine, Department of Pediatrics, University of Alabama at Birmingham, Birmingham, AL, USA
}

Previous studies have examined the quantitative results of using an organized approach of teaching and learning magic tricks - a therapeutic magic camp - but set aside the qualitative aspect. The purpose of this qualitative study is to explore the interpretation of the lived experience of children with hemiparesis after they have participated in a therapeutic magic camp. Six children, aged between 11 and 14 years old, with hemiparesis who completed the magic camp program 3 months prior were invited to participate in an in-person individual semistructured interview. The interviews were audio-recorded and transcribed verbatim for content analysis. Results from the initial qualitative analysis yielded categories of enjoyment, positive social and learning experience from participation in the camp, increased confidence, and self-motivation to use the affected upper limb. These categories were grouped into two themes: "having fun with others while learning" and "helping my arm perform." Having fun with others while learning was an amalgamation of fun, and positive social and learning experience. Helping my arm perform reflected a sense of increased competence and self-belief, and a willingness and motivation of the children to attempt challenging daily activities. The lived experiences of children with hemiplegia who participated in an organized delivery of learning to perform magic tricks yielded positive outcomes. These themes are consistent with the limited literature that is available suggesting that further research is needed to confirm the efficacy of the therapeutic magic camp intervention.

Keywords: Hemiplegia, Hand-arm bimanual intensive therapy, Motivation, Pediatrics, Magic tricks

\section{INTRODUCTION}

Hemiparesis is defined as partial paralysis or muscular weakness affecting one side of the body (Oskoui and Shevell, 2005). Children with hemiparesis experience movement limitations because of this weakness, spasticity or dystonia, and diminished range of motion lateralized to one side of the body, especially in the upper limb. These deficits result in impaired gross and fine motor coordination, leading to difficulties performing and completing bimanual tasks (Dong et al., 2017). Even though children with hemiparesis are eager to be proficient in motor performance, they often encounter frustration and failure in completing ordinary, everyday bimanual tasks (Skold et al., 2004). Of importance is the influence these physical limitations have on their levels of motiva- tion to perform tasks that require bimanual coordination (Miller et al., 2014). Motivation is a decisive factor in determining positive outcomes of motor performance and function for children with hemiparesis (Bartlett and Palisano, 2000). Compared to typically developing peers, children with hemiparesis have deep negative reactions to their inability to perform unimanual and bimanual tasks. As a result, they are often less motivated to persist with object-oriented task performance (Majnemer et al., 2010; Miller et al., 2014). Their motivation can be further diminished if they are presented only with simple and repetitive activities in therapy sessions which they may perceive as monotonous (Gilmore et al., 2010). Effective therapies are needed to improve the use and coordination of both hands/arms in these children when engaging in daily activities that require two hands to complete. Finding
${ }^{*}$ Corresponding author: Hon K. Yuen (D) https://orcid.org/0000-0001-5696-1845 Department of Occupational Therapy, School of Health Professions, University of Alabama at Birmingham, 1720 2nd Ave S, Birmingham, AL 35294, USA Email: yuen@uab.edu

Received: November 24, 2020 / Accepted: December 25, 2020
This is an Open Access article distributed under the terms of the Creative Commons Attribution Non-Commercial License (https://creativecommons.org/licenses/by-nc/4.0/) which permits unrestricted non-commercial use, distribution, and reproduction in any medium, provided the original work is properly cited. 
meaningful activities that connect with their interests, goals, and skills is vitally important to facilitate their motivation.

A systematic review of the literature found the benefits of artsbased interventions are becoming increasingly more evident in healthcare. One art form - the art of magic - has become especially popular as a therapeutic intervention for its potential to increase motivation, improve motor skills, and enhance confidence and resilience (Green et al., 2013; Hines et al., 2018). This approach is grounded in incentive theory of motivation, social learning theory, and motor learning theory (Killeen, 1982; Zwicker and Harris, 2009). Learning to perform magic tricks, especially in a group setting, has been shown to motivate children with disabilities, including those with hemiparesis, to use the affected upper limb for repetitive motor skills practice (Green et al., 2013; Hines et al., 2018). In these studies, a magic camp or magic club was formed that included participants with similar physical limitations and provided the opportunity for these children to engage in positive social experiences that promoted belonging and peer support (Green et al., 2013; Hines et al., 2018).

A therapeutic magic camp is one that incorporates the learning and performing of carefully selected magic tricks using an organized, theme-based approach (Green et al., 2013; Hines et al., 2019; Spencer et al., 2020). Studies using a therapeutic magic camp model have demonstrated beneficial effects on motor function improvements (unilateral and bilateral) in children with hemiparesis (Green et al., 2013; Hines et al., 2019; Spencer et al., 2020). At the conclusion of the magic club in the study of Hines et al. (2018), parents recognized that their children with hemiparesis had gained a feeling of increased competence and self-belief. This shift in thinking allowed for the development of a more autonomous attitude to attempt challenging daily activities.

While the physical benefits of a magic trick-based approach have been investigated, the psychosocial aspects have not. To refine the program elements-format, content and context, and appropriate evaluation instruments, it is important to listen to the voices of the children who have participated in a therapeutic magic camp program. The purpose of this study is to explore the lived experience of a child's participation in a therapeutic magic camp using the qualitative interview methodology.

\section{MATERIALS AND METHODS}

\section{Research design and ethical approval}

This study employed a qualitative research design using content analysis (Hsieh and Shannon, 2005). The Institutional Re-
Table 1. Demographics of child participants

\begin{tabular}{llllllc}
$\begin{array}{c}\text { Pseud- } \\
\text { onym }\end{array}$ & \multicolumn{1}{c}{ Diagnosis } & $\begin{array}{c}\text { Age } \\
(\mathrm{yr})\end{array}$ & Race & Gender & Grade & $\begin{array}{c}\text { Received UL } \\
\text { intensive therapy }\end{array}$ \\
\hline Amy & CP Left hemiparesis & 13.6 & White & Female & 8 th & 2 yr ago \\
Greta & CP Left hemiparesis & 13.5 & Black & Female & 8 th & 2 yr ago \\
Helen & CP Left hemiparesis & 10.6 & White & Female & 6 th & 0.5 yr ago \\
Oya & CP Left hemiparesis & 11.0 & Black & Female & 6 th & 0.5 yr ago \\
David & CP Right hemiparesis & 13.7 & White & Male & 8 th & $>5$ yr ago \\
Nick & $\begin{array}{l}\text { Right hemiparesis } \\
\text { (TBI): } 4.5 \text { yr ago }\end{array}$ & 11.9 & White & Male & 5 th & 2 yr ago \\
& & & & & \\
\hline
\end{tabular}

UL, upper limb; CP, cerebral palsy; TBI, traumatic brain injury.

Nick missed one day of the camp program due to medical appointment, received botulinum toxin injections during the 3-month follow-up period, and his parent/ guardian was not present while he was being interviewed.

view Board of the University of Alabama at Birmingham approved the study (protocol number: IRB-300001044).

\section{Participants}

Six of the seven children with hemiparesis, aged between 11 and 14 years old, who completed a magic camp program 3 months prior participated in an individual semistructured qualitative interview. Demographics (including status of hemiparesis and level of education) of the participants are shown in Table 1. Detailed eligibility criteria for children to be included in the magic camp program and recruitment procedures were reported elsewhere (Spencer et al., 2020).

\section{Procedures}

The magic camp program was designed as a 2-week intensive program for children and adolescents with hemiplegia to be held during the summer. Those who attended the 2-week camp experience participated in a total of $24 \mathrm{hr}$ of learning and practicing magic tricks in six sessions for a duration of $4 \mathrm{hr}$ each morning, 3 days a week, for 2 consecutive weeks. Each session consisted of intensive one-on-one hand-arm motor skills training (via practicing magic tricks) delivered by pairs of occupational therapy graduate students assigned to each child. Participants left the camp site at the completion of each morning session, and were encouraged to practice the selected magic trick at home. The goal of this magic camp was to engage children with hemiparesis to become more motivated to attempt bimanual daily activities using their affected hand/arm.

The protocol consisted of carefully selected magic tricks from the Magic Therapy manual (magictherapy.com), a resource offered to occupational therapists who wish to integrate magic tricks in a therapeutic setting. The magic tricks used in the camp were care- 
Table 2. Interview guiding questions to explore post magic camp experience

\begin{tabular}{|c|c|}
\hline Topic & Question \\
\hline $\begin{array}{l}\text { Reaction to magic camp } \\
\text { experience }\end{array}$ & $\begin{array}{l}\text { 1. How would you describe the magic camp experience? } \\
\text { 2. Describe what it was like to participate in the magic camp. } \\
\text { 3. What was it like to work with us through learning magic tricks? }\end{array}$ \\
\hline $\begin{array}{l}\text { Concerns, limitations, and } \\
\text { difficulties/barriers of the magic } \\
\text { camp and suggestions for } \\
\text { improvement }\end{array}$ & $\begin{array}{l}\text { 1. Describe the process of learning magic tricks. (Prompt level of frustration and fatigue) } \\
\text { 2. What do you like/dislike about the magic camp? } \\
\text { 3. Based on your experience, what were the difficulties you encountered in practicing magic tricks after the magic camp? } \\
\text { 4. How would you compare the magic camp experience with other summer camp? (Prompt relative advantages and/or drawbacks) } \\
\text { 5. What can we change to make the magic camp better? }\end{array}$ \\
\hline $\begin{array}{l}\text { Satisfaction, and usefulness of the } \\
\text { magic camp }\end{array}$ & $\begin{array}{l}\text { 1. How satisfied are you with the magic camp experience? } \\
\text { 2. How useful was learning the magic tricks? } \\
\text { 3. What was the most useful part about the magic camp to you? }\end{array}$ \\
\hline $\begin{array}{l}\text { Impacts on hand movement or daily } \\
\text { activities }\end{array}$ & $\begin{array}{l}\text { 1. Compared to before the magic camp, how do you feel about the use of your affected hand/arm? } \\
\text { 2. How would you describe the use of your affected hand/arm since your participation in the magic camp? } \\
\text { 3. How did the magic camp experience change your daily activities of your life? }\end{array}$ \\
\hline
\end{tabular}

fully selected, requiring the use of both hands in learning and performing the tricks. The unaffected/less affected hand was used to stabilize the prop and the more affected hand was be used to complete the movements necessary to accomplish the task. The same repertoire of magic tricks was taught to each participant using a systematic, scheduled approach. Samples of magic tricks have been reported elsewhere (Spencer et al., 2020).

Camp began with a daily warm-up activity, followed by practice on a specific magic trick under the guidance of each pair of occupational therapy students. Throughout the morning, participants were also given opportunities to use two hands performing daily activities such as snack preparation. Each day concluded with a group activity that included performances of magic tricks from campers and encouragement to continue practicing after each scheduled training session.

Participants' upper limb motor skills were assessed 3 times during the study, precamp, postcamp, and at a 3-month follow-up. An in-person qualitative individual interview with the participants was subsequently conducted at the 3-month follow-up evaluation. Capitalizing on the strong trust and rapport that had been established, the interview was directed by the same pair of occupational therapy students who taught the participants magic tricks during the camp. There were six pairs of student interviewers, and a pair of interviewers interviewed one participant. Participants (with the optional presence of their parents/guardians in the same room, but the questions were not directed to the parents/guardians) were asked open-ended questions designed to explore their camp experience and the impact of this experience on their daily life. Five of the six participants had one parent present.
Four parents would occasionally prompt their child to provide more details when answering the questions. They also added aspects of function they felt the child was able to do using their affected arm. Core questions of the interview guide are shown in Table 2. The interviewers largely followed the interview guide and the sequence of questions to facilitate discussion. However, flexibility in switching question order and including probes to elicit responses was implemented, depending on the unique flow of the interview interaction. The interviews were audio-recorded with the participants' consent and transcribed verbatim for content analysis. The core of the interview lasted an average of $7 \mathrm{~min}$. Transcripts were deidentified and the participants were given pseudonyms.

\section{Data analysis}

Using content analysis, categories related to the participants' experience with the magic camp and the impact of these experiences on their daily life were identified from the interview transcripts. The author (HKY) reviewed the transcripts multiple times, and coded key phrases and sentences through open coding (Vaismoradi et al., 2013), making sure the codes were aligned with the original texts to preserve the participant's meaning. HKY continued this process, comparing sections of text across all the interview transcripts, and, as new topics emerged, revised and added codes. These were then organized by similarity of content. Codes expressing similar concepts were grouped together to develop categories and themes.

An analyst triangulation approach was used to verify and validate the interpretation of data (Mitchell, 1986). Analyst triangu- 
lation facilitates a deeper understanding of the data and requires the use of several analysts to review the findings to eliminate selective perception and bias in an interpretive analysis (Mitchell, 1986). Two authors (KS and KK) familiar with the study coordinated with HKY to confirm and validate the data interpretation and confer about the meaning of the themes. By reviewing the transcripts and through discussion, a consensus was reached among the investigators. To further enhance credibility of the findings, an independent arbiter (LKV) with expertise in qualitative research, served as an external auditor to review the data categorization, interpretation, and conclusion (Whittemore et al., 2001). LKV was not involved in the original design of the study.

\section{RESULTS}

None of the participants had taken part in learning magic tricks prior to the study. Parents/guardians reported no changes in the participants' health, medication, and healthcare service utilization, except one child who had received botulinum toxin injections on his affected arm, during the 3-month follow-up period. None of the participants continued practicing magic tricks after the camp. Results from the initial qualitative analysis yielded categories of enjoyment, positive social and learning experience from participation in the camp, increased confidence, and self-motivation to use the affected upper limb. These categories were grouped into two themes: Having fun with others while learning and Helping my arm perform.

\section{Having fun with others while learning}

Participants all expressed that the camp was fun, and described the camp participation as an "awesome experience" (David), "enjoyed it a ton" (Nick), "excited about it" (Oya), "loved every second of it" (Amy), and "It was very fun and we got to meet new people." (Greta). Parents/ guardians confirmed these sentiments by expressing that their child enjoyed and was excited about coming to the camp every day. Some participants even asked whether they could come back and participate again next year.

In addition, participants had a chance to meet new people with shared physical and social similarities as well as occupational therapy students who were dedicated to conducting the camp. "It was a room full of people who had disabilities that were different than I. Which was cool to see but it was also really cool to participate. ...I got to like work with people [occupational therapy students] who were really dedicated, like I have been saying, but like it was also like a one-on-one personal experience that you just don't get with other types of summer camps." (David)

Participants also noted the magic camp was very different from their previous summer camp experiences in ways other than being fun and providing the opportunity to meet new people (both children with shared disability and occupational therapy students). They appreciated learning magic tricks. "I like that you get to learn magic tricks. I like that you get to learn the secret of the magic trick. You know when you see magicians and you never know, like, how they did it and now, like, after camp, I know more bow magic works." (Greta).

Participants were proud and confident as they showed the tricks that they learned to their family members. "... it felt really good to get up on a stage and perform in front of people I care about and other people that others cared about." (David). Other participants even attempted to teach their sibling the magic tricks.

\section{Helping my arm perform}

Participants perceived that practicing the magic tricks was helpful to their affected hand/arm and recognized their improvements. As one child, Greta, said, "After the magic camp, I think like my left hand was stronger than when we came. I think that my fingers bave more muscle." Another child, Nick, said, "It really helped my arm. It was very stiff (before the camp). (Now) it's way better. I'm using it a ton. ... Help me do more exercises at my physical education." When asked what she (Helen) can do with her affected arm now that she could not do before, she replied "raise it up."

The pride and confidence in using their affected hand/arm to complete daily activities was illustrated in the following excerpt between a participant, Oya, and her father:

Dad: Yeab well, whatever! How do you pick up something? You know like, bow you have to pick up your medicine? Don't you pick your medicine up better now?

Oya: Yeah, I guess.

Dad: She uses her left hand, with the medicine that she takes. And, it's self-motivated. We don't have to remind her, she does it on her own. So.

Oya: Oh, and scooping everything up with my left hand.

Dad: Well yes, ... What else do you use your left hand for?

Oya: like, bold on (yawns). A juice! A juice and picking things up for mommy. That's a good thing.

Dad: Do you use your left band to do it?

Oya: (sighs) Yes... daddy... You know what. Here's the best part daddy.

Finally, comments from the parents in the present study were almost identical to those provided by parents in the study of Hines et al. (2018). "That's more of a confidence thing but it's kind of nice to know that you have sometbing special you can do that maybe not 
everyone else can do." (Greta's mom).

\section{DISCUSSION}

As indicated from the qualitative interviews, participants not only valued the novelty of the magic camp, but also the social support from their peers with similar physical limitations and the dedicated student coaches, which motivated them not to miss any valuable magic learning lessons. These phenomena are summarized in the theme "Having fun with others while learning." The intense use of the affected hand/arm via learning and performing magic tricks resulted in a noticeable improvement in the participants' hand-arm motor function as was confirmed in previous studies (Green et al., 2013; Hines et al., 2019). The children discussed the change for the better in the use of their affected hand/ arm to perform daily activities with a sense of pride and confidence identified in the theme "Helping my arm perform." More importantly this feeling of self-belief and competence (i.e., "I can do it" attitude) was able to carry over even after the magic camp ended.

The power of the magic camp experience appeared to change participants' belief/confidence and motivation to use their affected hand/arm to attempt challenging daily activities, though none of the participants continued practicing magic tricks after the camp. The tricks are not cognitively challenging once the child knows the secret of successful performance. With more use of the affected hand, participants subconsciously and gradually self-initiated the use of their affected hand to perform various daily activities. Participant-generated descriptive information from the interviews supports the quantitative measure findings on upper limb motor function from the pilot study (Spencer et al., 2020).

A previous study explored parents' perspective of their child's participation in a magic club and the immediate impact of the magic club on their child with unilateral CP (Hines et al., 2018). The present study explored the impact of the magic camp from the perspective of the child participants. Interviews were conducted at 3-month follow-up instead of at the end of the magic camp event. Researchers deliberately interviewed the participants 3-month postcamp to learn whether there were any longer-term effects of the camp on their physical functioning. The study of Hines et al. (2018) was to explore how the parents of children with hemiparesis perceived their children's experience with the magic club. When the parents were interviewed, they interpreted their child's experience. In this study, children were directly asked to share their experience of the magic camp providing a unique, first-person response.

The themes that emerged from the child participants in this study were very similar to those generated from the parents of children who participated in the Amazing Magic Club study (Hines et al., 2018) — "the magic effect" and "I can do it." "The magic effect" theme embraced the children's enjoyment and appreciation of the specialness of the magic club. The "I can do it" theme embraced a sense of increased competence and self-belief, and willingness and motivation of the children to attempt challenging daily bimanual activities (Hines et al., 2018). The insights gathered on the magic camp experience from the children who participated may not have the same level of sophistication as their parents/guardians because of the expressive limitations of their age (11-14 years old) (Nippold, 2000). However, they did provide valuable insights on their lived camp experience. In fact, findings from the present study were consistent with the two themes identified in the previous study (Hines et al., 2018).

The findings of the current study indicate that the learning and performing of magic tricks is a beneficial and purposeful means of engaging children with hemiparesis to become more motivated to attempt bimanual daily activities using their affected hand/arm. These findings held true even 3 months after the completion of the magic camp program. The participants gained self-confidence or improvement in functioning by engaging in fun activities with peers. There are other summer camp programs for children with motor dysfunction without a theme-based approach that have also reported similar positive results in the psychosocial areas (Analytis et al., 2020; Zwicker et al., 2015).

The "I can do it" theme that emerged in the study of Hines et al. (2018) was reiterated by the participant, David, in the current study who stated, "Cool, I can do this now." The qualitative findings from the study of Hines et al. (2018) and the present study suggest that understanding the underlying psychological attributes (self-determination, self-motivation, and confidence) leading to the improvement in motor function should be included in future studies.

An important strength of this study is the inclusion of the voices of children with disabilities, which are often overlooked in research, to aid in the understanding of what may motivate them to engage in rehabilitation. The study of Hines et al. (2018) was designed to interview the parent and attempted to gain this information. Conversely, a limitation of this study is that the interview with the children was conducted only one time. Additional limitations may include findings may be biased by the social desirability responses from the participants because the same pair of 
student coaches who taught them magic tricks conducted the interview. However, their responses were consistent with the parents' report in the current study as well as in the literature (Hines et al., 2018). Because parents were present during the interviews, the possibility of social desirability bias regarding parents could also be a consideration. Children may have responded in ways they thought their parents wanted to hear; however, comments from the parents were rare because the questions were directed to the child, not the parent. The small sample size of this study may not achieve sampling saturation; however, the themes that emerged from the present study were congruent with those of a previous study involving perspectives from the parents of children with hemiparesis who participated in learning magic tricks (Hines et al., 2018), suggesting transferability of the findings. Finally, there was a participant whose motor dysfunction (i.e., hemiparesis) was due to traumatic brain injury at age 7.5 years old, but his perspectives on the magic camp are no different from other children with cerebral palsy.

\section{CONFLICT OF INTEREST}

No potential conflict of interest relevant to this article was reported.

\section{ACKNOWLEDGMENTS}

The authors received no financial support for this article.

\section{REFERENCES}

Analytis P, Warren N, Ponsford J. Supporting children and young people with an acquired brain injury (ABI) and their siblings: the experience of a camp for families with a child with an ABI. Neuropsychol Rehabil 2020 Feb 26:1-17. https://doi.org/10.1080/09602011.2020.1731556.

Bartlett DJ, Palisano RJ. A multivariate model of determinants of motor change for children with cerebral palsy. Phys Ther 2000;80:598-614.

Dong VA, Fong KN, Chen YF, Tseng SS, Wong LM. 'Remind-to-move' treatment versus constraint-induced movement therapy for children with hemiplegic cerebral palsy: a randomized controlled trial. Dev Med Child Neurol 2017;59:160-167.

Gilmore R, Ziviani J, Sakzewski L, Shields N, Boyd R. A balancing act: children's experience of modified constraint-induced movement therapy. Dev Neurorehabil 2010;13:88-94.

Green D, Schertz M, Gordon AM, Moore A, Schejter Margalit T, Farquharson Y, Ben Bashat D, Weinstein M, Lin JP, Fattal-Valevski A. A multi-site study of functional outcomes following a themed approach to hand-arm bimanual intensive therapy for children with hemiplegia. Dev Med Child Neurol 2013;55:527-533.

Hines A, Bundy AC, Black D, Haertsch M, Wallen M. Upper limb function of children with unilateral cerebral palsy after a magic-themed HABIT: a pre-post-study with 3- and 6-month follow-up. Phys Occup Ther Pediatr 2019;39:404-419.

Hines A, Bundy AC, Haertsch M, Wallen M. A magic-themed upper limb intervention for children with unilateral cerebral palsy: the perspectives of parents. Dev Neurorehabil 2018;22:104-110.

Hsieh HF, Shannon SE. Three approaches to qualitative content analysis. Qual Health Res 2005;15:1277-1288.

Killeen PR. Incentive theory: II. Models for choice. J Exp Anal Behav 1982; 38:217-232.

Majnemer A, Shevell M, Law M, Poulin C, Rosenbaum P. Level of motivation in mastering challenging tasks in children with cerebral palsy. Dev Med Child Neurol 2010;52:1120-1126.

Miller L, Ziviani J, Ware RS, Boyd RN. Mastery motivation in children with congenital hemiplegia: individual and environmental associations. Dev Med Child Neurol 2014;56:267-274

Mitchell ES. Multiple triangulation: a methodology for nursing science. ANS Adv Nurs Sci 1986;8:18-26.

Nippold MA. Language development during the adolescent years: aspects of pragmatics, syntax, and semantics. Top Lang Disord 2000;20: 15-28.

Oskoui M, Shevell MI. Profile of pediatric hemiparesis. J Child Neurol 2005;20:471-476

Skold A, Josephsson S, Eliasson AC. Performing bimanual activities: the experiences of young persons with hemiplegic cerebral palsy. Am J Occup Ther 2004;58:416-425.

Spencer K, Yuen HK, Jenkins GR, Kirklin K, Griffin AR, Vogtle LK, Davis D. Evaluation of a magic camp for children with hemiparesis: a pilot study. Occup Ther Health Care 2020:34:155-170.

Vaismoradi M, Turunen H, Bondas T. Content analysis and thematic analysis: Implications for conducting a qualitative descriptive study. Nurs Health Sci 2013;15:398-405.

Whittemore R, Chase SK, Mandle CL. Validity in qualitative research. Qual Health Res 2001;11:522-537.

Zwicker JG, Harris SR. A reflection on motor learning theory in pediatric occupational therapy practice. Can J Occup Ther 2009;76:29-37.

Zwicker JG, Rehal H, Sodhi S, Karkling M, Paul A, Hilliard M, Jarus T. Effectiveness of a summer camp intervention for children with developmental coordination disorder. Phys Occup Ther Pediatr 2015;35:163177. 